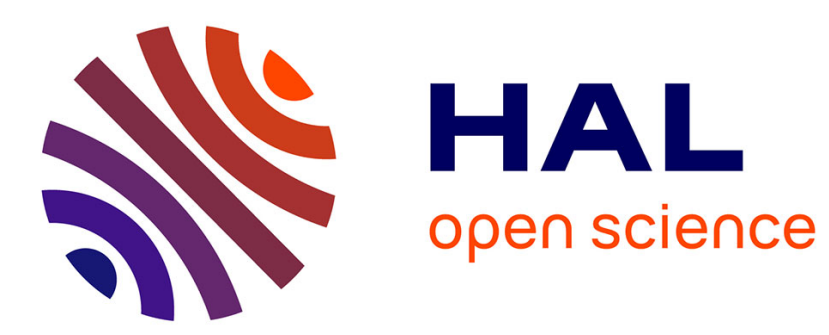

\title{
The singularity structure of fixed cycle directed site animals
}

\author{
J.A.M. Silva Duarte
}

\section{To cite this version:}

J.A.M. Silva Duarte. The singularity structure of fixed cycle directed site animals. Journal de Physique Lettres, 1985, 46 (12), pp.523-528. 10.1051/jphyslet:019850046012052300 . jpa-00232554

\section{HAL Id: jpa-00232554 https://hal.science/jpa-00232554}

Submitted on 1 Jan 1985

HAL is a multi-disciplinary open access archive for the deposit and dissemination of scientific research documents, whether they are published or not. The documents may come from teaching and research institutions in France or abroad, or from public or private research centers.
L'archive ouverte pluridisciplinaire HAL, est destinée au dépôt et à la diffusion de documents scientifiques de niveau recherche, publiés ou non, émanant des établissements d'enseignement et de recherche français ou étrangers, des laboratoires publics ou privés. 


\title{
LE JOURNAL DE PHYSIQUE-LETTRES
}

J. Physique Lett. 46 (1985) L-523 - L-528

15 JUIN 1985, PAGE L-523

Classification

Physics Abstracts

05.50

\section{The singularity structure of fixed cycle directed site animals}

\author{
J. A. M. Silva Duarte \\ Laboratório de Física Faculdade de Ciências, Universidade do Porto, 4000 Porto, Portugal
}

(Reçu le 8 février 1985, accepté le 23 avril 1985)

\begin{abstract}
Résumé. - La statistique des animaux dirigés sur un réseau, avec un nombre cyclomatique fixé, montre une structure de la singularité dominante parallèle à celle que l'on rencontre pour les animaux non dirigés avec un exposant $\theta_{c}=\theta_{0}-c$ pour un animal de nombre cyclomatique $c$. $\theta_{0}$, qui correspond à des animaux en arbre, est égal en toute dimension à l'exposant des animaux non dirigés.

Abstract. - Lattice data on fixed cycle directed animals show a structure for the dominant singularity paralleling that found for undirected animals, with an exponent $\theta_{c}=\theta_{0}-c$ for $c$-cycle animals. $\theta_{0}$, the value for trees is equal to the animal exponent in each dimension.
\end{abstract}

\section{Introduction and data derivation.}

As for normal animals the study of directed site animals with a fixed cyclomatic number is a topic of some interest.

Starting with trees or 0-cycle normal animals [1-3] various techniques have established a structure of dominant singularities for the generating function of such lattice animals

with

$$
A_{c}(x)=1+\sum_{n=1} A_{n, c} x^{n}
$$

and

$$
A_{n, c} \sim \lambda^{n} n^{-\theta_{c}}
$$

$$
\theta_{c}=\theta_{0}-c
$$

$n$-animal size, $\lambda$-tree multiplicity, $A_{n, c}$-total number of animals with size $n$ and $c$ cycles (loops).

Directed lattice animals have hardly been studied at all in this context, although exact results on total numbers of animals now include the dominant and subdominant singularities for dimensions 2 and 3, their connection in all dimensions to the value of the Yang-Lee edge singularity as well as some exact animal numbers $[4,5]$. The application of phenomenological renormalization to directed animals [6] remains an isolated exception on the study of cycles (in 2 dimen- 
sions) : the $\lambda$ in equation (1.2) for square site trees was obtained with the inherently high precision and both correlation exponents were shown to agree with those obtained for the complete set of directed animals, irrespective of cycle discriminations.

A series compendium of data on these cycle discriminations has become available in the literature [7] and in this paper we use this information to investigate the dimensional and cycle dependence of the dominant singularities for directed animals.

Most of the economies obtained through the combination of directed site animal nesting and computer cluster counting (for percolation and bond studies) have been referred in [7] and already briefly mentioned in [4] and [5]. In site directed animals the set of occupied sites on the normal hypersurface furthest from the origin acts as a source for the occupied sites on the next one and use of the recursion relations greatly reduces the amount of computerized counting.

In this study we have concentrated on the cyclomatic distributions for 2 and 3 dimensional cubic site animals already given in Duarte [7], and of which trees and 1-cycle animals are reprinted here for convenience with some discrete additions ( 2 terms in each case). As explained in [7] the lack of an overall percolation sum rule analogous to the one valid for cycle discrimination in normal animals (the so-called " bond expectancy rule » [2]), makes it convenient to use valence discriminations as a check on simple cycle discrimination. From Euler's law

$$
c=b-n+1
$$

where $b$ is the number of cluster bonds and

$$
b=\sum_{v=0}^{Z / 2} v n_{v}
$$

if $n_{v}$ is the number of animal sites with $v$ successor sites (hence measured through a kind of « outgoing valence "). If the animal discrimination is detailed to account for all possible outgoing

Table I. - Trees and 1-cycle site directed animals on the simple quadratic lattice.

\begin{tabular}{rrr}
\multicolumn{1}{r}{ Trees } & 1-cycle animals \\
1 & \multicolumn{2}{c}{1} \\
2 & 2 & \\
3 & 5 & \\
4 & 12 & 1 \\
5 & 29 & 6 \\
6 & 72 & 22 \\
7 & 182 & 70 \\
8 & 464 & 217 \\
9 & 1,190 & 662 \\
10 & 3,070 & 1,977 \\
11 & 7,958 & 5,814 \\
12 & 20,706 & 16,935 \\
13 & 54,045 & 48,954 \\
14 & 141,454 & 140,578 \\
15 & 371,119 & 401,504 \\
16 & 975,698 & $1,141,650$ \\
17 & $2,569,896$ & $3,233,992$ \\
18 & $6,779,948$ & $9,131,323$ \\
19 & $17,913,191$ & $25,710,308$
\end{tabular}


Table II. - Trees and 1-cycle site directed animals on the simple cubic lattice.

Trees

$\begin{array}{rr}1 & 1 \\ 2 & 3 \\ 3 & 12 \\ 4 & 49 \\ 5 & 204 \\ 6 & 870 \\ 7 & 3,787 \\ 8 & 16,722 \\ 9 & 74,643 \\ 10 & 336,108 \\ 11 & 1,524,438 \\ 12 & 6,956,214 \\ 13 & 31,905,603 \\ 14 & 146,985,960\end{array}$

1-cycle animals

$\begin{array}{rr}1 & \\ 2 & \\ 9 & 3 \\ 43 \\ 0 & 228 \\ 7 & 1,344 \\ 2 & 7,467 \\ 3 & 40,245 \\ 8 & 212,505 \\ 8 & 1,105,692 \\ 4 & 5,692,404 \\ 3 & 29,077,044 \\ 0 & 147,634,236\end{array}$

valence values (from $v=0$ to $Z / 2$ ) or all incoming valences (from $v=0, Z / 2$ ), or all total valences (the sum of the two), equations (1.4) and (1.5) are an alternative check on simple cycle studies. We have done this on all but the last s-cubic terms (for $n=14$ ). Of course the same economies apply to these extensive discriminations as to the complete set of directed animals.

\section{Numerical analyses.}

For the study of the asymptotic values of the dominant singularity and the multiplicity for fixed cycle animals we start by assuming algebraic singularities like in (1.2). Standard sequences to be investigated for $\lambda$ are the ratios

$$
\lambda_{n}=A_{n, \mathrm{c}} / A_{n-1, \mathrm{c}}
$$

and linear intercepts on them

$$
\lambda_{n}^{\prime}=n \lambda_{n}-(n-1) \lambda_{n-1}
$$

as well as their averages (when $\theta_{c}<0$ )

$$
\lambda_{n}^{\prime \prime}=\left(\lambda_{n}+\lambda_{n}^{\prime}\right) / 2
$$

and biassed sequences of the form

$$
\bar{\lambda}_{n}=n \lambda_{n} /\left(n-\theta_{c}\right)
$$

where $\theta_{c}$ is either known or conjectured from unbiassed sequences of the form

$$
\theta_{c}=n\left(1-\frac{\lambda_{n}}{\lambda_{n}^{\prime}}\right)
$$

In 2 dimensions both $\lambda_{n}$ and especially $\lambda_{n}^{\prime}$ converge rapidly towards the estimate for directed site trees by Nadal et al. [6], $\lambda=2.7126(1) \pm 1$. The uncertainty interval from the present series would be over 50 times greater. Using their central estimate instead of the linear intercepts in 


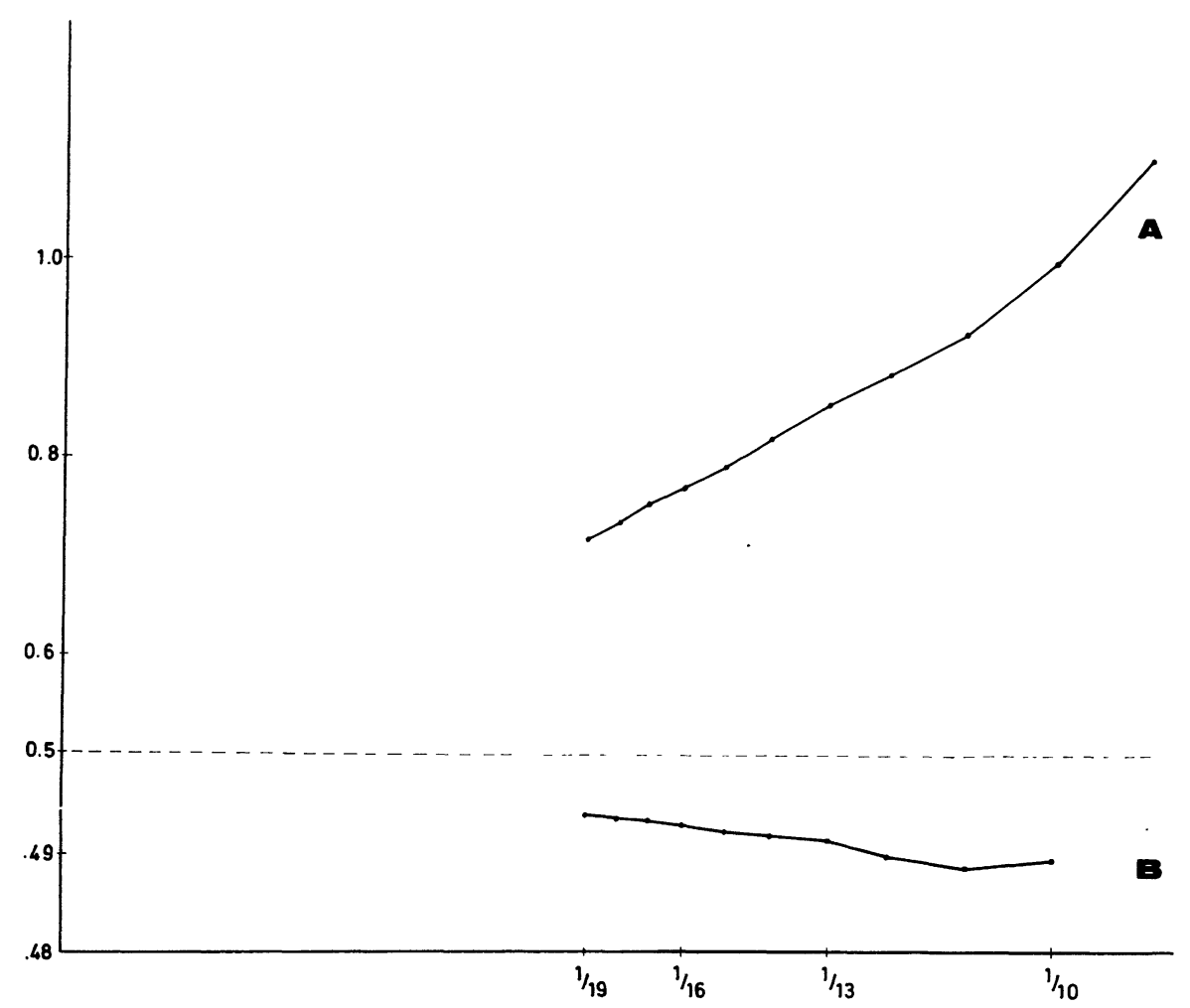

Fig. 1. - Estimates of $\theta_{0}$ (trees), line B and - $\theta_{1}$ (1-cycle animals), line A, plotted against the inverse size of directed site animals $(2 \mathrm{dim}$.).

(2.5) the $\theta_{0}(n)$ converge quite clearly to the exactly known animal value $\theta=1 / 2[4,5]$ (curve B in Fig. 1).

1-cycle animals show a good convergence pattern both for $\lambda$ and $\theta_{1}$. The exponent is negative so that averages like equation (2.3) between ratios and linear intercepts (or between ratios and biassed sequences $\bar{\lambda}_{n}$ (Eq. (2.4))), are weakly dependent on $n$ and in agreement with the tree multiplicity. Plotted in figure 1 are the $-\theta_{1}(n)$ sequences obtained with the central $\lambda$ value and the natural estimate is that

$$
\theta_{1}=\theta_{0}-1
$$

an exact counterpart of the singularity structure noticed in normal animals [3].

In 3 dimensions, simple cubic site trees give a reasonable pattern of convergence for unbiassed $\theta_{0}(n)$ sequences (plotted in Fig. 2, lines C). The exact animal value $\theta=5 / 6$ [4] is approached slowly both by this sequence and by linear intercepts on it.

The unbiassed sequence for $-\theta_{1}(n)$, obtained with $\lambda_{n}^{\prime \prime}$ instead of $\lambda_{n}^{\prime}$ in equation (2.5) is also shown in figure 2 , and while it shows a marked loss of accuracy with increasing cycle value, linear intercepts on them are levelling off rapidly and pointing inside an interval $-\theta_{1} \in[+0.05$, +0.25 ], compatible with the conjecture $\theta_{1}=-1 / 6$.

Comparable singularities are found in other 2 and 3 dimensional site animals (we have counted triangular, square matching and b.c.c. cycle animals to a smaller order of magnitude), while the higher cycle values [7] lead to a progressively poorer confirmation of the conjecture that here too, as for normal animals

$$
\theta_{c}=\theta_{0}-c
$$




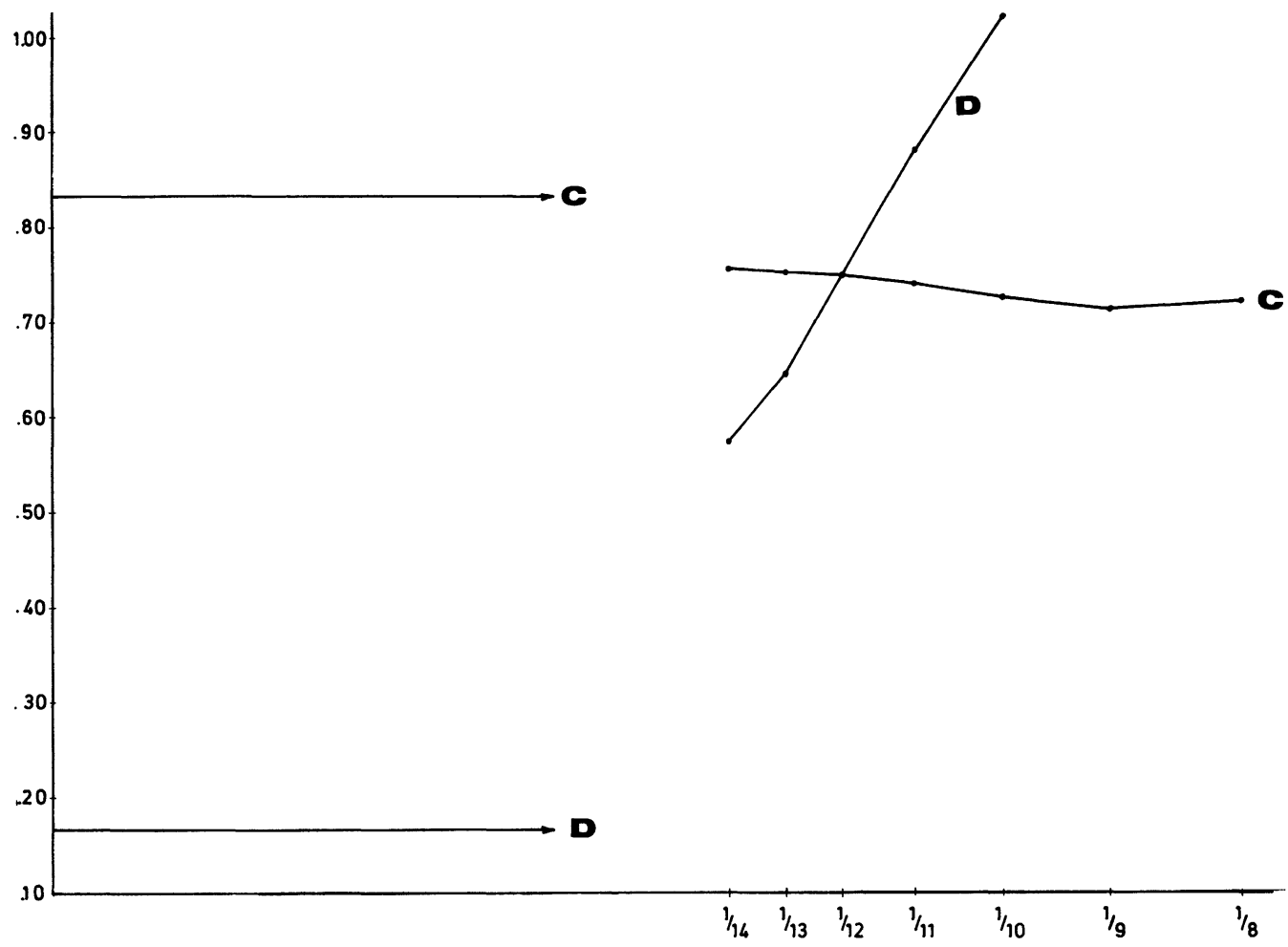

Fig. 2. - Estimates of $\theta_{0}$ (trees) line $\mathrm{C}$, with the exact animal value $\theta=5 / 6(\mathrm{C})$ and of $-\theta_{1}$ (1-cycle animals), line $\mathrm{D}$, with the conjecture $-\theta_{1}=1 / 6(\mathrm{D})$, plotted against the inverse size of directed site animals ( $3 \mathrm{dim}$.).

From global consideration of all the sequences for $\lambda$ from trees and 1-cycle animals we estimate in 3 dimensions

$$
\lambda=4.885 \pm 0.010 .
$$

The ratios $A_{n, c+1} / A_{n, c}$ furnish interesting information on the smaller of the confluences affecting both cycle values. From (2.7) and (1.2) these ratios should behave like $\alpha n+\beta n^{\sigma}(\sigma<1)$ and $\sigma \sim 0$ is reasonable from the smooth $d=2$ evidence on the present sequences. Alternative calculation of the confluence for $c=0$ (trees) is not in disagreement with a value $w=1$ (compatible with $\sigma=0$, above) despite a very irregular pattern of approach.

\section{Summary.}

Site directed animals show clearly a singularity structure characterized by progressively stronger dominant singularities, associated with an exponent dependence $\theta_{c}=\theta_{0}-c$ and starting from $\theta_{0}$ (the value for trees), which is the same as for the total number of directed animals in each dimension.

\section{Acknowledgments.}

The author is indebted to $\mathrm{N}$. Rivier for advice, to one of the referees for suggesting a reference to the ratios $A_{n, c+1} / A_{n, c}$ and to D. Dhar for correspondence, making available his simple quadratic enumerations. 
Note : after refereeing we became aware of Dhar's unpublished simple quadratic extension to $n=23$ (confirming table I and $A_{20,0}=47,390,540, A_{20,1}=72,213,064$ as meanwhile extended by us). Nadal et al. [6] $\lambda=2.7126(1) \pm 1$ remains unchallenged, while the confluent singularity can now be taken as $\sim 1$, as for the total number of animals [5].

\section{References}

[1] Lubensky, T. C. and Isaacson, J., Phys. Rev. A 20 (1979) 2130.

[2] Duarte, J. A. M. S. and Ruskin, H. J., J. Physique 42 (1981) 1585.

[3] Whittington, S. G., Torrie, G. M., Gaunt, D. S., J. Phys. A 16 (1983) 1695.

[4] Dhar, D., Phys. Rev. Lett. 51 (1983) 853.

[5] Dhar, D., Phani, N. K., Barma, M., J. Phys. A 15 (1982) L279.

[6] Nadal, J. P., Derrida, B., Vannimenus, J., J. Physique 43 (1982) 1561.

[7] Duarte, J. A. M. S., Portugal Phys. 15 (1984) 119. 